\title{
Velocity of Heart Rate Recovery in Post-Exercise Under Different Protocols of Active Recovery
}

\author{
Maria Angela Magalhaes De Queiroz Carreira, \\ Felipe Montes Pena, Mauricio B.F. Rachid, Leandro D. Tellerman, \\ Fabiana A Montaleone, Daniel C.A. Goncalves and Maria Eduarda D.C. Lobo
}

Department of Cardiology, Medicine School, Federal Fluminense University, Brazil

Received 2012-04-01, Revised 2012-08-04; Accepted 2013-11-12

\begin{abstract}
Abnormalities in the modulation of parasympathetic activity have been identified as a possible pathophysiological link with the association between decreases in heart rate recovery after exercise test. Hypothesis: To investigate if the application of different protocols of active recovery promotes difference in reducing Heart Rate (HR) in Post-Exercise (PE). This was a prospective, randomized trial of patients undergoing Exercise Testing (ET) and applied to two different protocols of active recovery. They were divided into G1 with recovery of $1.5 \mathrm{mph} / 2.5 \%$ and G2 $40 \%$ of the speed and slope of the peak in the PE. Variables were evaluated pre-test and intrinsic to the TE. We compared the recovery of FC 1 and 2 min in the PE. Statistical analysis used the chi-square test, Student's t test and considered $p<0.05$. We analyzed 939 patients and 852 selected for randomization. Were allocated to G1 403 patients with mean age $47.86 \pm 14.31$ years, $53.35 \%$ female and G2 with 449 patients with mean age $48.56 \pm 14.23$ years and 51.7\% were female. In the analysis of HR recovery of first $(22.00$ Vs. $23.57 ; p=0.315)$ and second $(36.17$ Vs. $37.70, p=0.06)$ minutes, G1 and G2 did not obtain differences. The use of different models of active recovery, applying fixed workload of $1.5 \mathrm{mph} / 2.5 \%$ slope or individually in $40 \%$ of the workload of the peak stress does not alter the recovery of $\mathrm{HR}$ in first or second minutes in the PE.
\end{abstract}

Keywords: Exercise, Autonomic System, Heart Rate

\section{INTRODUCTION}

The HR recovery after exercise represents the reactivation of parasympathetic activity and a reduction in sympathetic activity that typically occurs during the $30 \mathrm{sec}$ after exercise. Abnormalities in the modulation of parasympathetic activity have been identified as a possible pathophysiological link with the association between decrease in Heart Rate (HR) recovery after exercise test on a treadmill and increased mortality in the follow-up (Imai et al., 1994; Nishime et al., 2000).

Currently, the behavior of HR has been widely used in diagnostic analysis and prognosis of asymptomatic patients or with cardiovascular disease and prescription of exercise and control training (Lahiri et al., 2008). Researches in this field have studied the behavior of HR at rest, during exercise and recovery.

The rate of HR recovery proved to be prognostic in the first and second minute after the effort in populations referred for exercise testing than conventional, independent of exercise protocol employed (Cole et al., 1999; 2000). Since the first studies, Lauer et al. (2002) demonstrates conventionally that in using the active recovery protocols with treadmill speed of $1.5 \mathrm{mph}$ and $5 \%$ incline, the speed reduction would be considered satisfactory HR of $12 \mathrm{bpm}$ for the first minute and 22 $\mathrm{bpm}$ in the second minute. Several studies have proven the prognostic value of HR recovery on Tel: $552238243372-552181117099$ 
cardiovascular morbidity and mortality. Shetler et al. (2001) Nevertheless, all papers have considered the application of the methodology of active recovery (1.5 $\mathrm{mph} / 5 \%$ slope) for study validation (Ellestad, 2008). However, in our laboratory routinely employ active recovery in proportion to the workload peak $(40 \%$ of the speed and incline at peak exercise).

The aim of our study was to evaluate if different protocols of speed and incline of the treadmill the period of active recovery could influence the rate of $\mathrm{HR}$ reduction in post-exercise, promoting differences in the duration of inhibition of the sympathetic and parasympathetic activation.

\section{MATERIALS AND METHODS}

We evaluated 939 consecutive patients referred by their physician for TE and selected 852. Considering an alpha error of $5 \%$ and a beta error of $20 \%$, the sample size needed to identify the lowest correlation coefficient with statistical significance would be 402 patients in each group. We included patients over the age of eighteen and able to perform treadmill stress test. Exclusion criteria were: Contraindication to perform stress testing according to II Exercise Testing Guideline of the Brazilian Society of Cardiology (Andrade et al., 2002), did not have completed $2 \mathrm{~min}$ of active recovery phase, effort lasting less than $6 \mathrm{~min}$ arrhythmias and preventing the correct draft assessment of the HR. All patients underwent ET on treadmill ramp protocol with active recovery period post-exercise lasting at least 2 minutes and $30 \mathrm{sec}$ and kept under observation in passive recovery for at least 5 min. Patients were randomized before the start of the test, with Group one (G1) underwent active recovery at $40 \%$ speed and slope of peak exercise and in Group two (G2) was kept fixed speed of $1.5 \mathrm{mph}$ and absence of inclination.

\subsection{Exercise Test}

The maximum ET (symptom limited), was performed by a ramp protocol, according to the estimated functional capacity. Individuals under drug therapy have been tested on their medication regularly. Blood pressure was measured in an indirect way in mercury sphygmomanometer brand Wanros, fixed on a support within 1 meter of soil. During exercise blood pressure was measured and accomplished record of short electrocardiographic register with 13 derivations lead every $2 \mathrm{~min}$ at maximum effort in the immediate post-exercise $(10 \mathrm{sec})$ and 1, 2, 3 and $5 \mathrm{~min}$ of recovery and each event or change HR. The HR was determined automatically by the program for ergometry at rest, every 2 min of exercise, peak exercise and every minute during recovery. The measurements taken by the program at peak exercise and the first and second minute of recovery were checked manually at the end of the examination and corrected when necessary. The interruption of the exercise was done in accordance with the II Guidelines for Exercise Testing of the Brazilian Society of Cardiology (Andrade et al., 2002).

\subsection{Statistical Analysis}

The results were presented as frequencies (\%), mean and standard deviation. The chi-square and Student $\mathrm{t}$ test for independent samples were used for statistical evaluation of differences between proportions and means, respectively, after considering if the data were in line with the assumptions for these tests. The level of significance was $\mathrm{p}<0.05$.

\section{RESULTS}

The sample was evaluated for 852 patients with a mean age of $48.23+4.26$ years and $52.5 \%$ female. The G1 compound 403 patients with a mean age of $47.86 \pm 14.31$ years, $53.35 \%$ female and $4.5 \%$ were diabetic while the $\mathrm{G} 2$ consists of 449 patients with mean age $48.56 \pm 14.23$ years, $51.7 \%$ female and $5.1 \%$ had diabetes, both with similar percentages of female patients and diabetes mellitus. The characteristics of the groups are in Table 1.

Regarding the data evaluated in ET, there was no difference between HR pre-test between both groups $(80.03 \pm 13.98 \mathrm{Vs} 83.22 \pm 40.40, \mathrm{p}=0.132)$ and number of Metabolic Equivalents (METs) obtained at the end of the examination $(9.91 \pm 2.36$ Vs 9.69 $\pm 2.19, \mathrm{p}=0.164)$. (Table 2) There were no differences in the data intrinsic to the $\mathrm{TE}$ as the $\mathrm{FC}$ in the first minute of recovery postexercise between the G1 and G2 (23.0 Vs 22.57, p = 0.315 ) in the second minute of recovery (Vs 36.17. $37.70, \mathrm{p}=0.06)$ and VO2 $\max (39.19 \pm 5.7 \mathrm{Vs} 40.00 \pm 7.3$, $\mathrm{p}=0.38)$ (Fig. 1 and 2).

In the other variables in patients $<35$ years there was no difference between groups $(23.00 \pm 9.8$ Vs $24.05 \pm 8.2$, $\mathrm{p}=0.45)$ and those $>65$ years as HR first minute $(17.78 \pm 7.3 \mathrm{Vs} 17.87 \pm 8.2, \mathrm{p}=0.47)$ and second minute $(28.00 \pm 9.7$ Vs $26.77 \pm 10.01, p=0.24)$ recovery effort in the post and an average age between the groups (71.34 \pm 4.0 Vs $70.85 \pm 4.3, \mathrm{p}=0.24)$. 


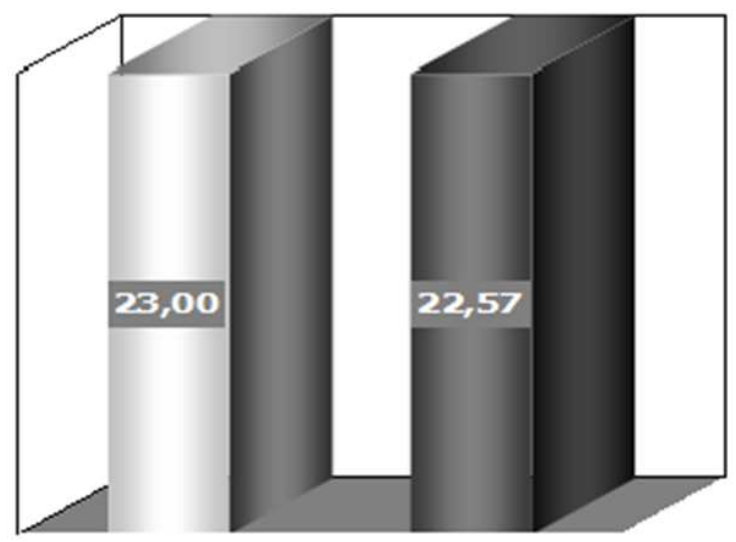

Fig. 1. Comparison between G1 and G2 in HR recovery at first minute post-exercise recovery

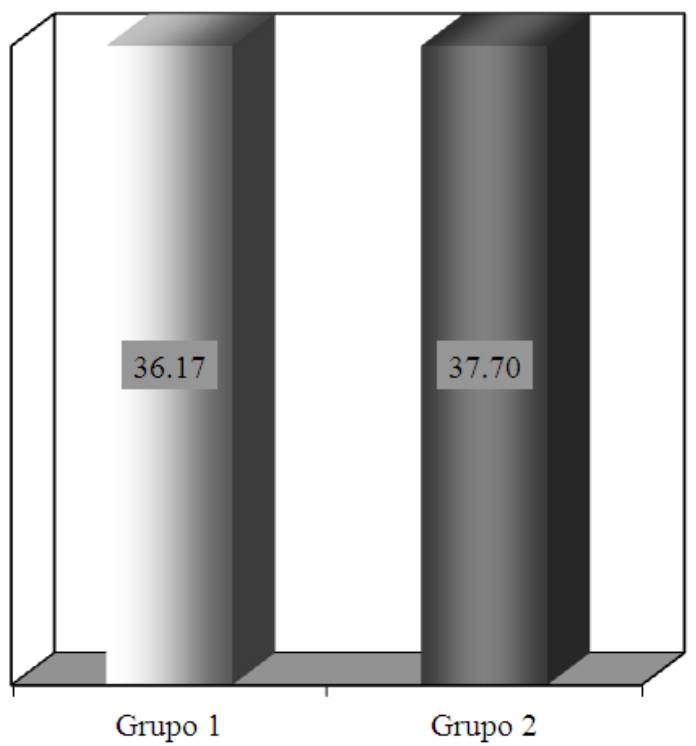

Heart rate recovery of second minute

$$
\mathrm{P}=0,06
$$

Fig. 2. Comparison between G1 and G2 in HR recovery at 2 minutes post-exercise recovery

Table 1. Characteristics of sample

\begin{tabular}{llll}
\hline & Group 1 & Group 2 & p. value \\
\hline $\mathrm{n}$ & 403 & 449 & \\
Age (years) & $47,86 \pm 14,31$ & $48,56 \pm 14,23$ & 0.48 \\
Male Sex (\%) & 53,35 & 51.67 & 0.63 \\
BMI (kg/m $\left.{ }^{2}\right)$ & $26,08 \pm 4,28$ & $26,18 \pm 4,04$ & 0.72 \\
Diabetes Melittus (\%) & 0.4 .46 & 0.5 .12 & 0.51 \\
Regular physical & 21.34 & 20.21 & 0.32 \\
activity (\%) & & & \\
Cardiac disease (\%) & 0.2 .32 & 0.1 .78 & 0.37 \\
\hline
\end{tabular}

Table 2. Comparative analysis between groups about intrinsic variables in ET

\begin{tabular}{llll}
\hline & Group 1 & Group 2 & p. value \\
\hline HR pre test (bpm) & $80,03 \pm 13,98$ & $83,22 \pm 40,40$ & 0.13 \\
$\begin{array}{l}\text { HR peak exercise } \\
\text { (bpm) }\end{array}$ & $155,74 \pm 22,47$ & $154,65 \pm 22,74$ & 0.48 \\
METs & $9,91 \pm 2,36$ & $9,69 \pm 2,19$ & 0.16 \\
$\begin{array}{l}\text { Exercise time (s) } \\
\text { Cronothropic }\end{array}$ & $519,84 \pm 83,03$ & $506,10 \pm 85,32$ & 0.41 \\
deficit (\%) & 0.9 .67 & 0.10 .29 & 0.33 \\
FAI (\%) & $-15,41 \pm 25,60$ & $-13,65 \pm 25,23$ & 0.31 \\
$\begin{array}{l}\text { Maximum } \\
\text { doubled-product }\end{array}$ & $27807 \pm 5796$ & $27943 \pm 5968$ & 0.73 \\
Ischemic (\%) & 0.5 .21 & 0.5 .34 & 0.45 \\
\hline
\end{tabular}

HR: Heart Rate; METs: Metabolic Equivalents; FAI: Functional Aerobic Impairment

\section{DISCUSSION}

In our study, no differences were observed between the recoveries of $\mathrm{HR}$ in post-exercise, regardless of active recovery protocol applied. As the HR recovery at first minute exercise depends basically on the return of parasympathetic autonomic activity, our results suggest no differences in the intensity of vagal activity, when applying speeds and different workloads in the period of active recovery.

During exercise, HR increase is due to increased sympathetic activity and decreased vagal activity (Cay, 2009). After cessation of exercise, the rapid decrease in $\mathrm{HR}$ is initially determined by vagal reactivation and subsequently by inhibition of sympathetic doing HR recovery a marker of cardiovascular parasympathetic activity (Schwartz et al., 1992). Therefore, the slow decline of HR after exercise is an indicator of malfunction of the ANS, specifically, the parasympathetic activity (Jouven et al., 2005). In our sample, the autonomic balance and cardiovascular system's ability to respond to withdrawal of sympathetic activity and beginning of the parasympathetic activity appear to have been affected when subjected to different methods of active recovery. When analyzed as extreme age $<35$ years old and $>65$ years old, both groups showed no differences when subjected to different workloads in active recovery.

Some studies (Gaibazzi et al., 2004; Kizilbash et al., 2006) have shown that the rate of HR recovery postexercise may be influenced by the type of ergometer. Ergometer in a reduction less than $22 \mathrm{bpm}$ has been associated with increased mortality. In the wake of these values can be influenced by the type of recovery applied, 
if passive, with abrupt cessation of movement, or active, with the belt still in motion for at least 2 min. Abrupt stopping of the treadmill determines a more rapid decrease in HR. According Halliwill et al. (2001), we can speculate that the presence of hypotension in the post stress can stimulate the arterial baroreflex vagal, bringing restoration and reducing the sympathetic activity (Halliwill et al., 1996). With the patient in the supine position immediately after exercise and during stress echocardiography, a reduction less than $18 \mathrm{bpm}$ at 1 min has been associated with higher morbimortality. Halliwill et al. (1996) with the abrupt interruption and the patient kept in orthostatic position, we can expect reductions of around $30 \mathrm{bpm}$ probably due to increased blood pressure by stimulating the parasympathetic activity (Perini and Veicsteinas, 2003). The type of exercise protocol employed, ramp or multistage during the exercise test on a treadmill, does not seem to influence the rate of HR recovery, since the protocol employed was appropriate to the functional condition of the individual. In our study, we excluded patients who arrived exhaustion less than 6 min of exercise, whereas the protocol applied in these cases was inappropriate and can result in different hemodynamic responses. However, for submaximal tests, Parekh and Lee (2005) suggest that exercise loads of $80 \% \mathrm{VO}_{2}$ reserve, result in major changes in parasympathetic bullet, when compared to exercise at $50 \% \mathrm{VO}_{2}$ reserve. Patients who have not reached the level of 10 fatigues by Borg scale of $1-10$ were excluded, existing therefore a mismatch between exercise intensity in both groups.

Concerning the type of active recovery protocol on a treadmill, since the early work comes up routinely using the recovery speed of $1.5 \mathrm{mph}$ with slope of $2.5 \%$ (Schwartz et al., 1992; Jouven et al., 2005; Lauer et al., 1996); in our laboratory we apply proportional reduction in the speed and incline at $40 \%$ maximum $\mathrm{VO}_{2}$ by considering that in populations with very high or very low functional capacity, recovery fixed at 1.5 $\mathrm{mph} / 2.5 \%$ is not adequate and can be very slow for an athlete race or too fast for the elderly or cardiac patients, which often reach a final speed of only two mph at peak effort, as in the Naughton protocol.

Our results show that in maximum treadmill exercise tests, with active recovery at a speed of 1.5 $\mathrm{mph} / 2.5 \%$ or with speed and incline to $40 \%$ of $\mathrm{VO}_{2}$ peak; we can use the same cutoffs as markers of risk: 12 bpm for $1 \mathrm{~min}$ and $22 \mathrm{bpm}$ for $2 \mathrm{~min}$ of recovery.

\section{CONCLUSION}

The use of different models of active recovery, applying fixed workload of $1.5 \mathrm{mph} / 2.5 \%$ slope or individually in $40 \%$ of the workload of the peak stress does not alter the recovery of heart rate in the first or in the second minute after exertion.

Although the hypothesis has been tested negative, this result is very important, since the demonstration that two different modes of active recovery did not influence the presence or absence of autonomic dysfunction, or even natural recovery of heart rate. More studies are necessary to assess the autonomic function in post-exercise, seeking to identify the mechanisms that determine the return of parasympathetic activity during recovery and its relation to cardiovascular disease and mortality.

\section{REFERENCES}

Andrade, J., F.S. Brito, F. Vilas-Boas, I. Castro and J.A. Oliveira et al., 2002. II diretrizes da sociedade brasileira de cardiologia sobre teste ergométrico. Arq. Bras. Cardiol., 78: 1-16. DOI: 10.1590/S0066782X2002000800001

Cay, S., 2009. Heart rate recovery after exercise: An important prognostic criterion. Int. J. Cardiol., 137: 187-188. DOI: 10.1016/j.ijcard.2009.05.005

Cole, C.R., E.H. Blackstone, F.J. Pashkow, C.E. Snader and M.S. Lauer, 1999. Heart-rate recovery immediately after exercise as a predictor of mortality. N. Engl. J. Med., 341: 1351-1357. DOI: 10.1056/NEJM199910283411804

Cole, C.R., J.M. Foody, E.H. Blackstone and M.S. Lauer, 2000. Heart rate recovery after submaximal exercise testing as a predictor of mortality in a cardiovascularly healthy cohort. Ann. Internal Med., 132: 552-555. DOI: 10.7326/0003-4819-132-7200004040-00007

Ellestad, M.H., 2008. Unconventional electrocardiographic signs of ischemia during exercise testing. Am. J. Cardiol., 102: 949-953. DOI: 10.1016/j.amjcard.2008.05.036

Gaibazzi, N., N. Petrucci and V. Ziacchi, 2004. Oneminute heart rate recovery after cycloergometer exercise testing as a predictor of mortality in a large cohort of exercise test candidates: Substantial differences with the treadmill-derived parameter. Ital Heart J., 5: 183-188. PMID: 15119500 
Halliwill, J.R., 2001. Mechanisms and clinical implications of post-exercise hypotension in humans. Exercise Sport Sci. Rev., 29: 65-70. DOI: 10.1097/00003677-200104000-00005

Halliwill, J.R., J.A. Taylor, T.D. Hartwig and D.L. Eckberg, 1996. Augmented baroreflex heart rate gain after moderate-intensity, dynamic exercise. Am. J. Physiol., 270: R420-R426. PMID: 8779874

Imai, K., H. Sato, M. Hori, H. Kusuoka and H. Ozaki et al., 1994. Vagally mediated heart rate recovery after exercise is accelerated in athletes but blunted in patients with chronic heart failure. J. Am. Coll Cardiol., 24: 1529-1535. DOI: 10.1016/07351097(94)90150-3

Jouven, X., J.P. Empana, P.J. Schwartz, M. Desnos and D. Courbon et al., 2005. Heart-rate profile during exercise as a predictor of sudden death. N Engl. J. Med., 352: 1951-1858. DOI: 10.1056/NEJMoa043012

Kizilbash, M.A., M.R. Carnethon, C. Chan, D.R. Jacobs and S. Sidney et al., 2006. The temporal relationship between heart rate recovery immediately after exercise and the metabolic syndrome: The CARDIA study. Eur. Heart J., 27: 1592-1596. DOI: 10.1093/eurheartj/ehl043

Lahiri, M.K., P.J. Kannankeril and J.J. Goldberger, 2008. Assessment of autonomic function in cardiovascular disease: Physiological basis and prognostic implications. J. Am. Coll. Cardiol., 51: 1725-1733. DOI: 10.1016/j.jacc.2008.01.038

Lauer, M.S., P.M. Okin and M.G. Larson et al., 1996. Impaired heart rate response to graded exercise: Prognostic implications of chronotropic incompetence in the framingham heart study. Circulation, 93: 15201526. DOI: $10.1161 / 01 . C I R .93 .8 .1520$
Lauer, M.S., S. Alexe, C.E. Pothier Snader, E.H. Blackstone and H. Ishwaran et al., 2002. Use of the logical analysis of data method for assessing longterm mortality risk after exercise electrocardiography. Circulation, 106: 685-690. PMID: 12163428

Nishime, E.O., C.R. Cole, E.H. Blackstone, F.J. Pashkow and M.S. Lauer, 2000. Heart rate recovery and treadmill exercise score as predictors of mortality in patients referred for exercise ECG. JAMA., 284: 1392-1398. DOI: 10.1001/jama.284.11.1392

Parekh, A. and M. Lee, 2005. Heart rate variability after isocaloric exercise bouts of different intensities. Med. Sci. Sports Exerc., 37: 599-605. DOI: 10.1249/01.MSS.0000159139.29220.9A

Perini, R. and A. Veicsteinas, 2003. Heart rate variability and autonomic activity at rest and during exercise in various physiological conditions. Eur. J. Applied Physiol., 90: 317-325. DOI: 10.1007/s00421-0030953-9

Schwartz, P.J., M.T. La Rovere and E. Vanoli, 1992. Autonomic nervous system and sudden cardiac death. Experimental basis and clinical observations for post-myocardial infarction risk stratification. Circulation, 85: I77-I91. PMID: 1728509

Shetler, K., R. Marcus, V.F. Froelicher, S. Vora and D. Kalisetti et al., 2001. Heart rate recovery: Validation and methodologic issues. J. Am. Coll. Cardiol., 38: 1980-1987. DOI: 10.1016/S0735-1097(01)01652-7 